\title{
Capecitabine Plus Oxaliplatin and Bevacizumab, Followed by Maintenance Treatment With Capecitabine and Bevacizumab for Patients Aged $>75$ Years With Metastatic Colorectal Cancer
}

\author{
Roberto Petrioli, ${ }^{1}$ Edoardo Francini, ${ }^{2}$ Sara Cherri, ${ }^{1}$ Pamela Torre, ${ }^{1}$ \\ Anna Ida Fiaschi, ${ }^{3}$ Salvatora Tindara Miano, ${ }^{1}$ Daniele Marrelli, ${ }^{4}$ Franco Rovello, ${ }^{4}$ \\ Guido Francini ${ }^{1}$
}

\begin{abstract}
The CAPOX/Bev (capecitabine plus oxaliplatin and bevacizumab) regimen has previously shown efficacy for patients with metastatic colorectal cancer (mCRC). Data on the efficacy and safety of CAPOX/Bev, followed by maintenance capecitabine and bevacizumab for elderly patients with mCRC, are limited. The present small study has shown that CAPOX/Bev, followed by maintenance capecitabine and Bev is safe and effective for mCRC patients aged $>75$ years.

Background: The aim of the present study was to evaluate the efficacy and safety of the combination of CAPOX-Bev (capecitabine [Cap] plus oxaliplatin and bevacizumab [Bev]), followed by maintenance Cap and Bev, for patients with metastatic colorectal cancer $(\mathrm{mCRC})$ and aged $>75$ years. Patients and Methods: The regimen consisted of intravenous oxaliplatin 130 to $100 \mathrm{mg} / \mathrm{m}^{2}$ on day 1 , oral Cap 750 to $1000 \mathrm{mg} / \mathrm{m}^{2}$ twice daily on days 1 to 14 , and Bev $7.5 \mathrm{mg} / \mathrm{kg}$ on day 1 , every 3 weeks. After 4 cycles of CAPOX-Bev, the patients without evidence of disease progression received maintenance treatment with Cap 1000 to $1250 \mathrm{mg} / \mathrm{m}^{2}$ twice daily on days 1 to 14 and Bev $7.5 \mathrm{mg} / \mathrm{kg}$ on day 1 , every 3 weeks, until disease progression or unacceptable toxicity. The primary endpoint was the 9-month disease control rate. Progression-free survival (PFS), overall survival (OS), and safety were the secondary endpoints. Results: Overall, 36 patients were enrolled from March 2012 to April 2017 at our institution. After completion of CAPOX/Bev, 15 patients (41.7\%) had a partial response, 18 (50.0\%) had stable disease, and $3(8.3 \%)$ had progressive disease. Thirty-three patients $(91.7 \%)$ received the Cap/Bev regimen as maintenance treatment for a median of 8.6 cycles (range, 3-14 cycles). The 9-month DCR was 58.3\% (95\% confidence interval [Cl], 40.8-74.5), the median PFS was 8.8 months ( $95 \% \mathrm{Cl}, 6.7-10.3$ months), and the median OS was 20.8 months ( $95 \% \mathrm{Cl}, 16.1-25.4$ months). With the CAPOX/Bev regimen, the most common grade 3 toxicity included neutropenia $(11.1 \%)$, diarrhea (5.5\%), nausea/ vomiting $(2.8 \%)$, and fatigue $(2.8 \%)$. Grade 3 neurotoxicity was not observed. With Cap/Bev maintenance therapy, grade 3 hand-foot syndrome was observed in 2 patients (6.0\%). Conclusion: CAPOX/Bev, followed by Cap/Bev as maintenance treatment, is safe and effective in terms of PFS and OS for elderly patients aged $>75$ years with mCRC.
\end{abstract}

Clinical Colorectal Cancer, Vol. m, No. m, a-m @ 2018 Published by Elsevier Inc.

Keywords: CAPEOX, Colon cancer, Elderly, mCRC, XELOX

R.P. and G.F. contributed equally to this work as first authors.

${ }^{1}$ Medical Oncology Unit, Department of Medicine, Surgery and Neurosciences,

University of Siena, Siena, Italy

${ }^{2}$ La Sapienza University, Rome, Italy

${ }^{3}$ Pharmacology Unit, Department of Medicine, University of Siena, Siena, Italy

${ }^{4}$ Department of General Surgery and Surgical Oncology, University of Siena, Siena, Italy
Submitted: Mar 21, 2018; Revised: Jun 28, 2018; Accepted: Jul 2, 2018

Address for correspondence: Roberto Petrioli, MD, Medical Oncology Unit, Department of Medicine, Surgery and Neurosciences, University of Siena, Viale Bracci, Siena 53100, Italy E-mail contact: r.petrioli@ao-siena.toscana.it 


\section{CAPOX/Bev and XELOX for $\mathrm{mCRC}$}

\section{Introduction}

Colorectal cancer (CRC) is the third most common malignancy and the fourth leading cause of cancer mortality worldwide. ${ }^{1}$ More than one third of CRC diagnoses occur in patients aged $\geq 75$ years. $^{2}$ Nevertheless, clinical trials designed to include the elderly population are still limited, as is the evidence supporting the treatment of advanced CRC for this specific population. ${ }^{3}$

Despite surgical resection currently representing the only chance of long-term cure, a variable proportion of patients develop recurrence or present at diagnosis with locally advanced or metastatic CRC (mCRC) and thus are candidates for systemic chemotherapy. Of the available chemotherapy agents, the fluoropyrimidines 5fluorouracil (5-FU) and capecitabine (Cap) are the most active and conventionally administered drugs for mCRC. ${ }^{4}$ In the past, large randomized trials demonstrated that oxaliplatin (L-OHP) combined with 5-FU (FOLFOX) or Cap (CAPOX) and the antiangiogenic agent bevacizumab (Bev) significantly improved progression-free survival (PFS) and overall survival (OS) for patients with mCRC. ${ }^{5,6}$ In most analyses, FOLFOX or CAPOX, plus Bev were administered for $\geq 12$ or 8 cycles, respectively, or until progressive disease or unacceptable toxicity developed. Grade 3/4 hematologic and nonhematologic adverse events occur frequently. In particular, peripheral sensory neuropathy (PSN), a cumulative dose-limiting toxicity of L-OHP, often requires drug discontinuation before disease progression. ${ }^{5-7}$ Alternative treatment strategies, such as "stop and go" or intermittent chemotherapy, have been evaluated to minimize the severity of adverse events. ${ }^{8,9}$ A phase II study led by our research group showed promising results in terms of PFS and a very low incidence of grade 3 PSN for mCRC patients treated with FOLFOX stop and go and Cap as maintenance chemotherapy. ${ }^{10}$ In addition, the activity of FOLFOX or CAPOX, plus Bev, followed by leucovorin/5-FU or CAP with Bev as maintenance treatment was reported in other recent studies. ${ }^{11,12}$ However, in most trials, the population included was aged $<75$ years and had a good performance status (PS), on average; hence, data suggesting the most effective chemotherapy regimen for elderly and vulnerable patients are limited. Older adults with mCRC often have comorbidities that complicate the administration of aggressive treatments; thus monochemotherapy or best supportive care alone are often recommended for patients aged $>75$ years. However, elderly patients with a good PS and considered fit using a comprehensive geriatric assessment might benefit from the administration of a multidrug treatment regimen. ${ }^{13}$

The present study evaluated the clinical efficacy and tolerability of induction chemotherapy with CAPOX/Bev followed by Cap/Bev as maintenance treatment in nonfrail patients aged $>75$ years.

\section{Patients and Methods Eligibility Criteria}

For the present prospective observational study, patients aged $>75$ years with histologically proven and radiologically measurable mCRC were enrolled at our institution. No previous chemotherapy for advanced disease was allowed. Adjuvant chemotherapy, if administered, had to have been completed $\geq 6$ months before study entry. The other eligibility criteria included Eastern Cooperative Oncology Group PS of 0 to 2, a life expectancy of $\geq 3$ months, adequate hematologic parameters (absolute neutrophil count, $\geq 1.5 \times 10^{9} / \mathrm{L} ;$ platelet count, $\left.\geq 100 \times 10^{9} / \mathrm{L}\right)$, creatinine $<1.5$ times the upper limit of the normal range, total bilirubin $<3$ times the upper normal limit, aspartate and alanine aminotransferase $<3$ times the upper normal limit, and the absence of a second primary tumor other than nonmelanoma skin cancer or in situ cervical carcinoma. The exclusion criteria were histologic evidence of predominantly squamous cell cancer, a history of gross hemoptysis $(\geq 2.5 \mathrm{~mL}$ ), brain metastases or previous treatment of brain metastasis, uncontrolled pleural or pericardial effusion or ascites, pregnancy or lactation, a history of documented hemorrhagic diathesis or coagulopathy, therapeutic anticoagulation, the regular use of aspirin (>325 mg/d), nonsteroidal anti-inflammatory agents known to inhibit platelet function, radiation therapy within 21 days before enrollment or major surgery within 28 days before enrollment, clinically significant cardiovascular disease, and medically uncontrolled hypertension.

Using a baseline geriatric assessment that included evaluation of activities of daily living, instrumental activities of daily living, and the Charlson score for comorbidities, ${ }^{14}$ the patients were classified as fit, vulnerable, or frail. Specifically, subjects with activities of daily living limitations and $>2$ comorbidities were classified as frail and were excluded from the present study. ${ }^{15}$ All the included patients provided their written informed consent.

\section{Patient Evaluation}

Patients were seen at the start of every chemotherapy cycle for a physical examination, monitoring of symptoms and toxic effects, assessment of renal function, and a complete blood count. mCRC was reassessed after completion of 4 cycles of CAPOX/Bev and thereafter at 3-month intervals until evidence of disease progression (PD). The objective tumor response was evaluated using the Response Evaluation Criteria in Solid Tumors, version 1.1.

\section{Treatment Delivery}

Induction chemotherapy consisted of 4 cycles of intravenous L-OHP 100 to $130 \mathrm{mg} / \mathrm{m}^{2}$ on day 1 plus oral Cap 750 to 1000 $\mathrm{mg} / \mathrm{m}^{2}$ twice daily on days 1 to 14 and intravenous Bev $7.5 \mathrm{mg} / \mathrm{kg}$ on day 1 every 3 weeks. Given the advanced age of our study population, L-OHP and Cap were administered at a full-dose solely to fit patients aged $\leq 80$ years and at a reduced dose $\left(100 \mathrm{mg} / \mathrm{m}^{2}\right.$ for L-OHP and $750 \mathrm{mg} / \mathrm{m}^{2}$ twice daily for Cap) to vulnerable patients and subjects aged $>80$ years to minimize the occurrence of L-OHP- and Cap-related adverse events. Maintenance treatment consisted of Cap $1250 \mathrm{mg} / \mathrm{m}^{2}$ twice daily on days 1 to 14 (1000 $\mathrm{mg} / \mathrm{m}^{2}$ twice daily for vulnerable patients) and Bev at a dose of $7.5 \mathrm{mg} / \mathrm{kg}$.

\section{Toxicity}

Toxicity was assessed using the National Cancer Institute Common Terminology Criteria for Adverse Events, version 4.02. Treatment was delayed if, on the planned day of treatment, the neutrophil count was $<1500 / \mathrm{mm}^{3}$, the platelet count was $<100,000 / \mathrm{mm}^{3}$, or the patient had persistent diarrhea or stomatitis of grade $>1$. Any patient who required $>2$ weeks for recovery from adverse reactions was excluded from the present study. In the event of grade 4 hematologic or any other severe 
Roberto Petrioli et al

(grade $\geq 3$ ) organ toxicity, the chemotherapeutic drug doses were reduced by $25 \%$ for subsequent courses.

\section{Statistical Analysis}

The primary endpoint was the 9-month disease control rate (DCR), defined as the percentage of patients with stable disease (SD) or partial (PR) or complete response within 9 months after the start of induction treatment. Fluoropyrimidines can be considered reference drugs for elderly patients with advanced CRC, and most studies investigating these agents as monochemotherapy have suggested that $\sim 20 \%$ of patients will still be progression free at 9 months after treatment onset. ${ }^{4}$ The hypothesis for the present study was that using CAPOX/Bev as induction chemotherapy and Cap/ Bev as maintenance treatment $\geq 40 \%$ of patients would be progression free 9 months after the start of chemotherapy. We calculated that a total of 33 patients should be recruited to yield an $80 \%$ probability to correctly select the treatment when it was superior by an absolute difference of $20 \%$ in the 9-month DCR (Simon's minimax design). ${ }^{16}$

The secondary endpoints included safety, PFS (calculated as the time from the first chemotherapy infusion to PD, death from any cause, or censored at the last follow-up visit, whichever came first), OS (measured from the date of treatment start to the date of death or the last follow-up visit), and the objective response rate (the proportion of patients achieving a PR or complete response). The Kaplan-Meier method was used to evaluate the distributions of PFS and OS, including the median time-to-event and $95 \%$ confidence intervals (CI). Statistical analyses were conducted using STATAiC software.

\section{Results}

\section{Patient Characteristics}

From March 2012 to April 2017, 36 patients with newly diagnosed mCRC who were aged $>75$ years were enrolled in the present study. The baseline demographic and clinical characteristics of patients are presented in Table 1 . Their median age was 79 years (range, 76-87 years), and more than one third (13 of 36; 36.1\%) of the patients were aged $>80$ years. The vast majority of patients ( 33 of 36; 91.7\%) had an Eastern Cooperative Oncology Group PS of 0 or 1 and approximately two thirds ( 24 of $36 ; 66.7 \%$ ) of the patients were classified as fit. Twenty-eight patients $(77.7 \%)$ had undergone previous surgery with curative intent and were offered our treatment protocol after documentation of unresectable locally advanced or metastatic disease. Sixteen patients (44.4\%) had previously received adjuvant chemotherapy. Twenty-six patients (72.2\%) had liver metastases. At baseline, all the patients had $\geq 1$ comorbidity, and most (23 of 36; 63.9\%) had > 1 (Table 2). The most frequent concomitant illnesses were cardiovascular disease (16 of $36 ; 44.4 \%)$.

\section{Efficacy}

All 36 enrolled patients completed 4 cycles of induction chemotherapy and were evaluable for response and toxicity. The starting LOHP dose was $130 \mathrm{mg} / \mathrm{m}^{2}$ for 17 fit patients aged $\leq 80$ years and 100 $\mathrm{mg} / \mathrm{m}^{2}$ for 12 vulnerable patients and 7 fit patients aged $\geq 80$ years. Of the 36 patients, 15 (41.7\%) had a PR and 18 (50.0\%) had SD as the best response to therapy after initial CAPOX/Bev (Table 3). The 33 patients $(91.7 \%)$ with a PR or SD after induction chemotherapy
Table 1 Patient Characteristics $(n=36)$

\begin{tabular}{|c|c|}
\hline Characteristic & n (\%) \\
\hline \multicolumn{2}{|l|}{ Age, y } \\
\hline Median & 79 \\
\hline Range & $76-87$ \\
\hline Patients aged $>80 \mathrm{y}$ & $13(36.1)$ \\
\hline \multicolumn{2}{|l|}{ Gender } \\
\hline Male & $22(61.1)$ \\
\hline Female & $14(38.9)$ \\
\hline \multicolumn{2}{|l|}{ ECOG PS } \\
\hline 0 & $14(38.9)$ \\
\hline 1 & $19(52.8)$ \\
\hline 2 & $3(8.3)$ \\
\hline \multicolumn{2}{|l|}{ Geriatric assessment } \\
\hline Fit & $24(66.7)$ \\
\hline Vulnerable & $12(33.3)$ \\
\hline \multicolumn{2}{|c|}{ Charlson comorbidity index } \\
\hline Median & 1 \\
\hline Range & $0-2$ \\
\hline \multicolumn{2}{|l|}{ Primary tumor } \\
\hline Colon & $27(75.0)$ \\
\hline Right & $7(19.4)$ \\
\hline Left & $20(55.5)$ \\
\hline Rectum & $9(25.0)$ \\
\hline \multicolumn{2}{|l|}{ Primary tumor resection } \\
\hline Colon & $21(58.3)$ \\
\hline Rectum & $7(19.4)$ \\
\hline \multicolumn{2}{|l|}{ KRAS status } \\
\hline Mutated & $23(63.9)$ \\
\hline Wild type & $13(36.1)$ \\
\hline \multicolumn{2}{|l|}{ Metastatic sites } \\
\hline Liver & $26(72.2)$ \\
\hline Lymph nodes & $10(27.8)$ \\
\hline Peritoneum & $7(19.4)$ \\
\hline Lung & $9(25.0)$ \\
\hline Other & $3(8.3)$ \\
\hline$>1$ Metastasis & $14(38.9)$ \\
\hline \multicolumn{2}{|c|}{ Primary adjuvant chemotherapy } \\
\hline No & $20(55.5)$ \\
\hline Yes & $16(44.4)$ \\
\hline
\end{tabular}

Abbreviations: $E C O G=$ Eastern Cooperative Oncology Group; PS = performance status.

with CAPOX/Bev received maintenance chemotherapy with Cap and Bev for a median of 8.6 cycles (range, 3-14 cycles). The objective response rate for Cap/Bev maintenance treatment was $15 \%$. Twentyseven patients discontinued maintenance chemotherapy because of PD. For 7 of these patients, who were fit and aged $\leq 80$ years, L-OHP was reintroduced. One patient was lost to follow-up 10 months after the onset of induction chemotherapy. One patient was continuing maintenance treatment at the last follow-up visit. The 9-month DCR was $58.3 \%$ (95\% CI, 40.8\%-74.5\%), and median PFS was 8.8 months (95\% CI, 6.7-10.3 months). Finally, 21 patients (58.3\%) 


\section{CAPOX/Bev and XELOX for mCRC}

\section{Table 2 Patient Comorbidities}

\begin{tabular}{l|c}
\hline Comorbidities & Patients, n (\%) \\
Cardiovascular & $16(44.4)$ \\
\hline Hypertension & $12(33.3)$ \\
\hline Coronary artery disease & $5(13.9)$ \\
\hline Arrhythmia & $4(11.1)$ \\
\hline Diabetes mellitus & $5(13.9)$ \\
\hline Dyslipidemia & $5(13.9)$ \\
\hline Respiratory & $8(22.2)$ \\
\hline Genitourinary & $4(11.1)$ \\
$>1$ Comorbidity & $23(63.9)$ \\
\hline
\end{tabular}

received irinotecan-based chemotherapy as second-line treatment, and 9 patients $(25 \%)$ received regorafenib as third-line therapy.

At a median follow-up period of 31.5 months (range, 7.3-41.9 months), 28 patients had died, and the median OS was 20.8 months (95\% CI, 16.1-26.4 months). The PFS and OS for the 36 enrolled patients are illustrated in Figure 1.

\section{Treatment Toxicity}

The toxicity occurring during the $\mathrm{CAPOX} / \mathrm{Bev}$ induction regimen is summarized in Table 4. Grade 3 adverse events included neutropenia (11.1\%), diarrhea (5.5\%), nausea/vomiting (2.8\%), and fatigue $(2.8 \%)$. Four patients $(11.1 \%)$ required $\geq 1 \mathrm{~L}-\mathrm{OHP}$ and Cap dose reductions because of toxicity, and 13 cycles (9.0\%) were delayed for $\geq 1$ week.

Toxicity during maintenance treatment was mild. Two patients (6.0\%) experienced grade 3 hand-foot syndrome, one $(3.0 \%)$ developed grade 3 diarrhea, one (3.0\%) had grade 3 hypertension, and one (3.0\%) developed grade 3 venous thrombosis (Table 5). No patient experienced grade 3 proteinuria or epistaxis. A dose reduction of Cap was required for 4 patients. Bev administration was definitively interrupted for 1 patient aged 83 years 3 months after the onset of maintenance treatment because of the occurrence of cognitive impairment. The neurologic symptoms, after neurologic examination and encephalic magnetic resonance imaging, was attributed to prolonged exposure to Bev, and the patient completely recovered $\sim 6$ weeks after Bev discontinuation.

\begin{tabular}{l|c}
\hline Table 3 Results & $\mathbf{n ~ ( \% )}$ \\
\hline Variable & $0(0)$ \\
Objective response & $15(41.6)$ \\
\hline \multicolumn{1}{c|}{ Complete response } & $18(50.0)$ \\
\hline Partial response & $3(8.3)$ \\
\hline Stable disease & $58.3(40.8-74.5)$ \\
\hline Progressive disease & $8.8(6.7-10.3)$ \\
\hline 9-mo DCR, \% (95\% Cl) & $20.8(16.1-25.4)$ \\
\hline PFS, mo (95\% Cl) & \\
\hline OS, mo (95\% Cl)
\end{tabular}

Abbreviations: $\mathrm{Cl}=$ confidence interval; $\mathrm{DCR}=$ disease control rate; $\mathrm{OS}=$ overall survival; $\mathrm{PFS}=$ progression-free survival.
Figure 1 Progression-Free Survival (Solid Line) and Overall Survival (Dashed Line) for 36 Enrolled Patients

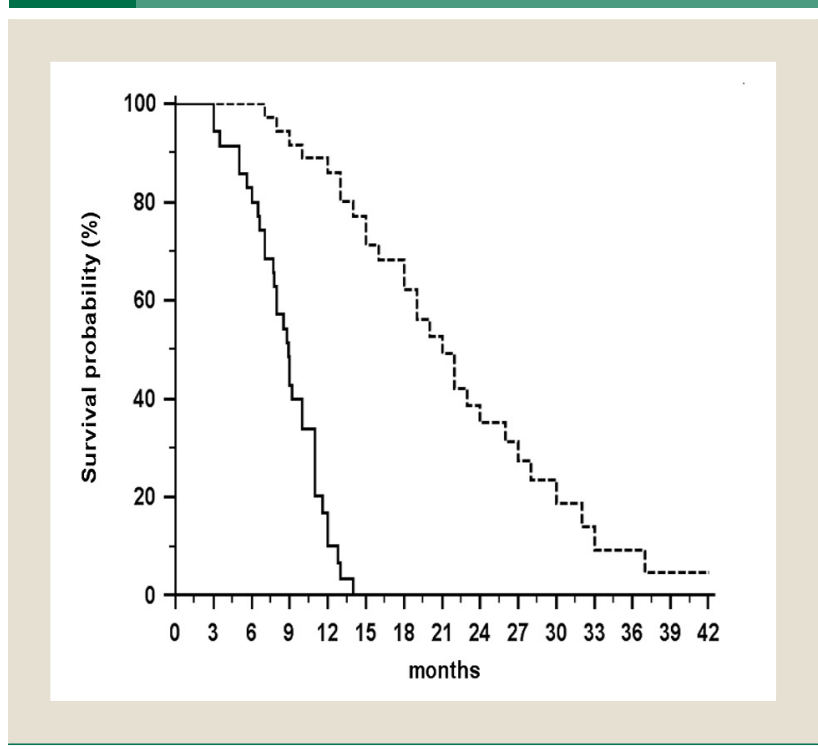

\section{Discussion}

Although favorable results have been reported previously for the addition of Bev to standard first-line chemotherapy for mCRC patients, increased toxicity has also been observed in association with this combination; thus, particular caution should be exercised when using it for the treatment of elderly patients. ${ }^{17} \mathrm{~A}$ reasonable approach for nonfrail patients aged $>75$ years with $\mathrm{mCRC}$ seems to be an initial short-term combination chemotherapy regimen, followed by a fluoropyrimidine associated with a biologic agent as maintenance therapy. ${ }^{18,19}$ To the best of our knowledge, the

\section{Table 4 Adverse Events With CAPEOX/Bev}

\begin{tabular}{l|c|c}
\multirow{2}{*}{$\begin{array}{l}\text { Adverse Event } \\
\text { Hematologic }\end{array}$} & \multicolumn{2}{|c}{ Grade, n (\%) } \\
\cline { 2 - 3 } Neutropenia & $\mathbf{2}$ & $\mathbf{3}$ \\
\hline Anemia & $5(15.1)$ & $4(11.1)$ \\
\hline Thrombocytopenia & $4(11.1)$ & $1(2.8)$ \\
\hline Nonhematologic & $5(15.1)$ & $0(0)$ \\
\hline Nausea & & \\
\hline Vomiting & $3(8.3)$ & $1(2.8)$ \\
\hline Fatigue & $1(2.8)$ & $0(0)$ \\
\hline Stomatitis & $8(22.2)$ & $1(2.8)$ \\
\hline Sensory neuropathy & $3(8.3)$ & $0(0)$ \\
\hline Diarrhea & $9(25.0)$ & $0(0)$ \\
\hline Constipation & $7(19.4)$ & $2(5.5)$ \\
\hline Hypertension & $8(22.2)$ & $1(2.8)$ \\
\hline Proteinuria & $6(16.7)$ & $0(0)$ \\
\hline Epistaxis & $1(2.8)$ & $0(0)$ \\
\hline Cognitive & $0(0)$ & $0(0)$ \\
\hline
\end{tabular}

No grade 4 events developed in any of the 36 patients.

Abbreviations: Bev = bevacizumab; CAPEOX = capecitabine, oxaliplatin. 


\begin{tabular}{|c|c|c|}
\hline $\begin{array}{ll}\text { Table } 5 & \text { Adverse E } \\
\text { Treatment }\end{array}$ & $\mathrm{Fap} / \mathrm{BeI}$ & \\
\hline \multirow[b]{2}{*}{ Adverse Event } & \multicolumn{2}{|c|}{ Grade, n (\%) } \\
\hline & 2 & 3 \\
\hline \multicolumn{3}{|l|}{ Cap-associated } \\
\hline Neutropenia & $2(6.0)$ & $0(0)$ \\
\hline Anemia & $2(6.0)$ & $0(0)$ \\
\hline Thrombocytopenia & $1(3.0)$ & $0(0)$ \\
\hline Nausea/vomiting & $1(3.0)$ & $0(0)$ \\
\hline Abdominal pain & $3(9.0)$ & $0(0)$ \\
\hline Diarrhea & $3(9.0)$ & $1(3.0)$ \\
\hline Hand-foot syndrome & $3(9.0)$ & $2(6.0)$ \\
\hline \multicolumn{3}{|l|}{ Bev-associated } \\
\hline Hypertension & $3(9.0)$ & $1(3.0)$ \\
\hline Proteinuria & $2(6.0)$ & $0(0)$ \\
\hline Epistaxis & $2(6.0)$ & $0(0)$ \\
\hline Cognitive & $1(6.0)$ & $0(0)$ \\
\hline Venous thrombosis & $2(6.0)$ & $1(3.0)$ \\
\hline
\end{tabular}

Abbreviations: Bev = bevacizumab; Cap = capecitabine.

present study is the first to suggest that CapOX/Bev for 4 cycles, followed by $\mathrm{Cap} / \mathrm{Bev}$ as maintenance treatment, is feasible and active for mCRC patients aged $>75$ years. Albeit with the limitations of a small study, the 58.3\% 9-month DCR, 8.8-month PFS, and 20.8-month median OS compare well with the results commonly reported with the most active combination chemotherapy regimens in younger populations. ${ }^{5,6}$ The results of the present study are also in line with the best results previously observed in patients aged $>65$ years with mCRC receiving CAPOX/Bev or FOLFOX/Bev. ${ }^{20,21}$ A recent study demonstrated a 74.4\% DCR, 7.9-month PFS, and 20.1-month OS for elderly patients with mCRC receiving Bev combined with modified CAPOX for a maximum of 12 cycles. $^{22}$ However, most of those patients were aged $<75$ years, but all our patients were aged $\geq 75$ years. Thus, although the median OS of 20.8 months in the present study might seem lower than expected, especially with the use of the newer agents available for patients with mCRC, $36.1 \%$ of our enrolled patients were aged $>80$ years ( 5 were aged $>85$ years), and $38.9 \%$ of our patients had $>1$ metastatic site, including $19.4 \%$ of the patients with peritoneum involvement. In addition, the DCR was only $38 \%$ with irinotecan-based chemotherapy as second-line treatment, and only 4 of 13 patients with wild-type tumors could receive anti-EGFR agents (7 had a primary right tumor). Finally, regorafenib, which could be administered as third-line therapy for only 9 patients owing to a poor PS, resulted in a median PFS of 4.3 months.

In addition, the findings of our analysis are similar to those reported in recent studies that highlighted the role of oral fluoropyrimidines combined with $\mathrm{Bev}$ as maintenance therapy after initial induction combination chemotherapy. ${ }^{23,24}$ In particular, the large randomized trial CAIRO 3 (Maintenance Treatment Versus Observation After Induction in Advanced Colorectal Carcinoma) reported a greater median PFS than that observed in the present study (11.7 vs. 8.8 months, respectively) and similar median OS
(21.6 vs. 20.8 months, respectively) for the CAP/Bev maintenance group after 6 cycles of initial CAPOX/Bev. Although the CAIRO 3 trial included patients aged $\leq 81$ years, the median age of the 279 subjects enrolled in the maintenance group was only 63 years. ${ }^{23}$ Furthermore, a recent randomized phase III study reported an OR of 66.7, a PFS of 8.3 months, and an OS of 23.8 months for mCRC patients treated with CAPOX plus Bev for 6 cycles, followed by $\mathrm{Cap} / \mathrm{Bev}$ as maintenance treatment. The median age of that study population was 56 years and in our study, was 79 years. ${ }^{25}$ Compared with the present analysis, better results have been recently reported for a group of 52 Japanese patients with mCRC treated with a stop and go strategy of CAPOX and Bev for 6 cycles, followed by Cap/Bev as maintenance (OR, 55.8; PFS, 12.4 months; OS, 30.6 months). However, only a few patients were aged $>75$ years, and the median age was 66 years. Moreover, grade 3 PSN was observed in 2 patients $(3.8 \%)$, probably resulting from the planned reintroduction of $\mathrm{L}-\mathrm{OHP}{ }^{26}$

Although favorable results with the combination of Bev and Cap as maintenance therapy have also been reported previously, ${ }^{23,24}$ to the best of our knowledge, our analysis is the first to enroll patients aged $>75$ years. Furthermore, other maintenance approaches have been previously tested in mCRC. Bev alone used as maintenance treatment after induction chemotherapy did not improve survival compared with no treatment (observation; median OS, 21.7 vs. 22.0 months) in a recent randomized phase III trial. ${ }^{27}$ In contrast, single-agent capecitabine might be considered an appropriate maintenance treatment option after XELOX or FOLFOX induction regimens for mCRC patients with acceptable toxicities. ${ }^{18}$ Capecitabine alone as maintenance treatment resulted in longer median OS in a group of 136 patients (25.6 months) compared with that for the observation-only group (23.3 months). However, the result was not statistically significant $(P=.2247)$. Furthermore, the median age of the Cap-treated cohort (56 years) was much lower than that of our study population (79 years). ${ }^{18}$

Despite the advanced median age and potential frailty of our study population, the treatment was well tolerated and no unexpected toxic effects developed in the present study. The starting dose of L-OHP and Cap was for many patients lower than the conventional starting dose of $130 \mathrm{mg} / \mathrm{m}^{2}$ and $2000 \mathrm{mg} / \mathrm{m}^{2}$, respectively. However, such dose reductions were determined owing to the high rates of patients classified as vulnerable $(33.3 \%)$ or presenting with $>1$ concomitant comorbidities (63.9\%) in our analysis. Possibly also for this reason, grade 3 neutropenia occurred in only $11.1 \%$ of patients, and the severity of nausea and vomiting was mild during initial CAPOX/Bev. In contrast, PSN, which is related to the cumulative L-OHP dose, developed in several patients in the present study (grade 2 in 25\%) but never reached grade 3. This safety profile is similar to, or even better than, that usually reported with conventional CAPOX or FOLFOX-4 combined with Bev for younger patients and could be partly attributed to the shorter exposure to L-OHP, the accurate selection of nonfrail patients, and the starting dose reductions of L-OHP and Cap. Thus, although no consensus has yet been reached regarding the definition of frailty status, we deemed that patient age $<85$ years, the ability to perform activities of daily living, $<3$ concomitant comorbidities, and no cognitive impairment were the factors that can best determine a nonfrail patient. A reasonable approach that could be applied 


\section{CAPOX/Bev and XELOX for $\mathrm{mCRC}$}

by practicing clinicians could be the use at baseline of a screening tool such as the G8 questionnaire, which allows for a quick distinction between fit and nonfit patients. Nonfit subjects could be further evaluated using a comprehensive geriatric assessment, which can discriminate vulnerable and frail patients. ${ }^{28,29}$

The toxicities associated with $\mathrm{Cap} / \mathrm{Bev}$ during maintenance treatment were consistent with the known adverse events associated with these drugs, especially for long-term use, and were grade 1 to 2 in most cases. However, a good safety profile is often the main advantage of a treatment strategy that includes maintenance treatment after induction chemotherapy before PD. The proportion of grade $3 \mathrm{Cap} / \mathrm{Bev}$-related adverse events in the present study was lower than that reported in the AVEX clinical trial [a study of bevacizumab (Avastin) in combination with capecitabine (Xeloda) in elderly patients with metastatic colorectal cancer], which evaluated Cap/Bev versus Cap alone for patients aged $>70$ years with $\mathrm{mCRC}$ (hand-foot syndrome, $6 \%$ vs. $16 \%$; diarrhea, $3 \%$ vs. $7 \%$; venous thromboembolic events, $3 \%$ vs. $8 \%$, respectively). ${ }^{30}$ This difference might have simply resulted from the small size of the present study and/or better selection of the enrolled patients (in the AVEX trial, patients were not considered fit for combination chemotherapy). Finally, Bev was interrupted in 1 patient aged $>80$ years owing to the development of cognitive impairment. Although the findings from the present study and other reports suggest that appropriately selected older patients can tolerate well the addition of Bev to chemotherapy, caution should be observed for patients aged $>80$ years, who are commonly at risk of developing cognitive decline, with consequent deterioration of their quality of life. ${ }^{31}$

The potential shortcomings of our study included the single-arm design, relatively small sample size, and long study duration of 5 years. The latter was chiefly the consequence of the singleinstitution experience that included only very elderly patients, which clearly delayed recruitment. Although clinical trials are warranted to confirm our results in a larger population, our data could aid treatment decision making for nonfrail patients aged $>75$ years with mCRC.

\section{Conclusion}

The results of the present study suggest that CAPOX/Bev, followed by Cap/Bev as maintenance treatment, is safe and active as first-line treatment for nonfrail mCRC patients aged $>75$ years and can achieve encouraging results in PFS and OS.

\section{Clinical Practice Points}

- In the past, several studies showed the efficacy of FOLFOX or CAPOX, plus Bev, induction chemotherapy, followed by 5fluorouracil/leucovorin or $\mathrm{Cap} / \mathrm{Bev}$ as maintenance treatment, for patients with mCRC.

- However, most studies included patients aged $<75$ years with good PS.

- Therefore, data on the efficacy and safety of this regimen for elderly patients with mCRC are limited.

- Also, because patients aged $>75$ years commonly present with comorbidities, monochemotherapy or best supportive care have typically been preferred.
- In the present prospective observational study, we reported the efficacy and tolerability of CAPOX/Bev as induction treatment, followed by $\mathrm{Cap} / \mathrm{Bev}$ as maintenance therapy, for 36 patients with newly diagnosed $\mathrm{mCRC}$ aged $>75$ years and screened as nonfrail.

- On completion of the CAPOX/Bev regimen, more than one third of the patients had a PR, one half had SD, and only few had developed disease progression.

- Nearly all patients received the Cap/Bev regimen as maintenance treatment.

- The 9-month DCR, median PFS, and median OS compared well with those observed in several studies evaluating similar regimens for younger populations.

- During CAPOX/Bev regimen and Cap/Bev maintenance treatment, grade 3 adverse events were not common, in particular, grade 3 neurotoxicity was not observed.

- Therefore, CAPOX/Bev, followed by maintenance Cap/Bev, can be safely proposed as first-line treatment for nonfrail patients with $\mathrm{mCRC}$ aged $>75$ years.

\section{Disclosure}

The authors declare that they have no competing interests.

\section{References}

1. Arnold M, Sierra MS, Laversanne M, Soerjomataram I, Jemal A, Bray F. Global patterns and trends in colorectal cancer incidence and mortality. Gut 2017; 66: 683-91.

2. Young PE, Womeldorph CM, Johnson EK, et al. Early detection of colorectal cancer recurrence in patients undergoing surgery with curative intent: current status and challenges. J Cancer 2014; 5:262-71.

3. SEER Cancer Statistics Review, 1975-2013, based on November 2015 SEER data submission, posted to the SEER web site, April 2016. Bethesda, MD: National Cancer Institute, Available at: https://seer.cancer.gov/csr/1975_2013/. Accessed March 19, 2018.

4. Van Cutsem E, Twelves C, Cassidy J, et al. Oral capecitabine compared with intravenous fluorouracil plus leucovorin in patients with metastatic colorectal cancer: results of a large phase III study. J Clin Oncol 2001; 19:4097-106.

5. Saltz LB, Clarke S, Díaz-Rubio E, et al. Bevacizumab in combination with oxaliplatin-based chemotherapy as first-line therapy in metastatic colorectal cancer: a randomized phase III study. J Clin Oncol 2008; 26:2013-9.

6. Hochster HS, Hart LL, Ramanathan RK, et al. Safety and efficacy of oxaliplatin and fluoropyrimidine regimens with or without bevacizumab as first-line treatment of metastatic colorectal cancer: results of the TREE study. J Clin Oncol 2008; 26:3523-9.

7. Grothey A. Oxaliplatin-safety profile: neurotoxicity. Semin Oncol 2003; 30 (4 Suppl 15):5-13.

8. Maindrault-Goebel F, Tournigand C, André T, et al. Oxaliplatin reintroduction in patients previously treated with leucovorin, fluorouracil and oxaliplatin for metastatic colorectal cancer. Ann Oncol 2004; 15:1210-4.

9. Wadhawan A, Stephens R, Adams R. Intermittent therapy in the palliative treatment of metastatic colorectal cancer. Expert Rev Anticancer Ther 2009; 9:125-34.

10. Petrioli R, Paolelli L, Marsili S, et al. FOLFOX-4 stop and go and capecitabine maintenance chemotherapy in the treatment of metastatic colorectal cancer. Oncology 2006; 70:345-50.

11. Vaidyanathan G, Groman A, Wilding G, Fakih MG. Stop and go FOLFOX plus bevacizumab chemotherapy in the first-line treatment of metastatic colorectal cancer. Oncology 2010; 79:67-71.

12. Díaz-Rubio E, Gómez-España A, Massutí B, et al. Group for the treatment of digestive tumors: first-line XELOX plus bevacizumab followed by XELOX plus bevacizumab or single-agent bevacizumab as maintenance therapy in patients with metastatic colorectal cancer: the phase III MACRO TTD study. Oncologist 2012; $17: 15-25$.

13. Pallis AG, Papamichael D, Audisio R, et al. EORTC Elderly Task Force experts opinion for the treatment of colon cancer in older patients. Cancer Treat Rev 2010; 36:83-90.

14. Repetto L, Fratino L, Audisio RA, et al. Comprehensive geriatric assessment adds information to Eastern Cooperative Oncology Group performance status in elderly cancer patients: an Italian Group for Geriatric Oncology study. J Clin Oncol 2002; 20:494-502.

15. Balducci L, Extermann M. Management of cancer in the older person: a practical approach. Oncologist 2000; 5:224-37. 
16. Simon R. Optimal two-stage designs for phase II clinical trials. Control Clin Trials 1989; 10:1-10.

17. Jain VK, Hawkes EA, Cunningham D. Integration of biologic agents with cytotoxic chemotherapy in metastatic colorectal cancer. Clin Colorectal Cancer 2011; 10:245-57.

18. Luo HY, Li YH, Wang W, et al. Single-agent capecitabine as maintenance therapy after induction of XELOX (or FOLFOX) in first-line treatment of metastatic colorectal cancer: randomized clinical trial of efficacy and safety. Ann Oncol 2016 27:1074-81.

19. Esin E, Yalcin S. Maintenance strategy in metastatic colorectal cancer: a systematic review. Cancer Treat Rev 2016; 42:82-90.

20. Kabbinavar FF, Hurwitz HI, Yi J, Sarkar S, Rosen O. Addition of bevacizumab to fluorouracil-based first-line treatment of metastatic colorectal cancer: pooled analysis of cohorts of older patients from two randomized clinical trials. J Clin Oncol 2009; 27:199-205.

21. Kozloff MF, Berlin J, Flynn PJ, et al. Clinical outcomes in elderly patients with metastatic colorectal cancer receiving bevacizumab and chemotherapy: results from the BRiTE observational cohort study. Oncology 2010; 78:329-39.

22. Vamvakas L, Matikas A, Karampeazis A, et al. Capecitabine in combination with oxaliplatin and bevacizumab (AXELOX) as 1st line treatment for fit and vulnerable elderly patients (aged $>70$ years) with metastatic colorectal cancer (mCRC): a multicenter phase II study of the Hellenic Oncology Research Group (HORG) BMC Cancer 2014; 14:277.

23. Simkens LH, van Tinteren H, May A, et al. Maintenance treatment with capecitabine and bevacizumab in metastatic colorectal cancer (CAIRO3): a phase 3 randomised controlled trial of the Dutch Colorectal Cancer Group. Lancet 2015; 385:1843-52.
24. Hegewisch-Becker S, Graeven U, Lerchenmüller CA, et al. Maintenance strategies after first-line oxaliplatin plus fluoropyrimidine plus bevacizumab for patients with metastatic colorectal cancer (AIO 0207): a randomised, non-inferiority, open-label, phase 3 trial. Lancet Oncol 2015; 16:1355-69.

25. Yalcin S, Uslu R, Dane F, et al. Bevacizumab + capecitabine as maintenance therapy after initial bevacizumab + XELOX treatment in previously untreated patients with metastatic colorectal cancer: phase III "stop and go" study results—a Turkish Oncology Group Trial. Oncology 2013; 85:328-35.

26. Nakayama G, Ishigure $\mathrm{K}$, Yokoyama $\mathrm{H}$, et al. The efficacy and safety of CapeOX plus bevacizumab therapy followed by capecitabine plus bevacizumab maintenance therapy in patients with metastatic colorectal cancer: a multi-center, single-arm, phase II study (CCOG-0902). BMC Cancer 2017; 17:243.

27. Aparicio T, Ghiringhelli F, Boige V, et al. Bevacizumab maintenance versus no maintenance during chemotherapy-free intervals in metastatic colorectal cancer: a randomized phase III trial (PRODIGE 9). J Clin Oncol 2018; 36:674-81.

28. Wildiers H, Heeren P, Puts M, et al. International Society of Geriatric Oncology consensus on geriatric assessment in older patients with cancer. J Clin Oncol 2014; 32:2595-603.

29. Soubeyran P, Bellera C, Goyard J, et al. Screening for vulnerability in older cancer patients: the ONCODAGE prospective multicenter cohort study. PLoS One 2014; 9:e115060.

30. Cunningham D, Lang I, Marcuello E, et al, AVEX Study Investigators. Bevacizumab plus capecitabine versus capecitabine alone in elderly patients with previously untreated metastatic colorectal cancer (AVEX): an open-label, randomised phase 3 trial. Lancet Oncol 2013; 14:1077-85.

31. Mohile SG, Magnuson A. Comprehensive geriatric assessment in oncology. Interdiscip Top Gerontol 2013; 38:85-103. 